\title{
Severity assessment in maximally treated ICH patients
}

The max-ICH score

Jochen A. Sembill, MD

Stefan T. Gerner, MD

Bastian Volbers, MD

Tobias Bobinger, MD

Hannes Lücking, MD

Stephan P. Kloska, MD

Stefan Schwab, MD

Hagen B. Huttner, MD

Joji B. Kuramatsu, MD

Correspondence to

Dr. Kuramatsu:

joji.kuramatsu@uk-erlangen.de

\section{ABSTRACT}

Objective: As common prognostication models in intracerebral hemorrhage (ICH) are developed variably including patients with early $(<24$ hours) care limitations (ECL), we investigated its interaction with prognostication in maximally treated patients and sought to provide a new unbiased severity assessment tool.

Methods: This observational cohort study analyzed consecutive ICH patients $(n=583$ ) from a prospective registry over 5 years. We characterized the influence of ECL on overall outcome by propensity score matching and on conventional prognostication using receiver operating characteristic analyses. We established the max- $\mathrm{ICH}$ score based on independent predictors of 12month functional outcome in maximally treated patients and compared it to existing models.

Results: Prevalence of ECL was 19.2\% ( $n=112 / 583$ ) and all of these patients died. Yet propensity score matching displayed that $50.7 \%(n=35 / 69)$ theoretically could have survived, with $18.8 \%$ ( $n=13 / 69$ ) possibly reaching favorable outcome (modified Rankin Scale score 0-3). Conventional prognostication seemed to be confounded by ECL, documented by a decreased

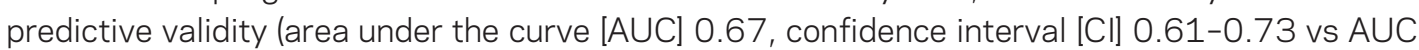
$0.80, \mathrm{Cl} 0.76-0.83 ; \mathrm{p}<0.01$ ), overestimating poor outcome (mortality by $44.8 \%$, unfavorable outcome by $10.1 \%$ in maximally treated patients. In these patients, the novel max-ICH score (0-10) integrates strength-adjusted predictors, i.e., NIH Stroke Scale score, age, intraventricular hemorrhage, anticoagulation, and ICH volume (lobar and nonlobar), demonstrating improved predictive accuracy for functional outcome (12 months: AUC 0.81, Cl 0.77-0.85; $p<0.01$ ). The max- $-\mathrm{CH}$ score may more accurately delineate potentials of aggressive care, showing favorable outcome in $45.4 \%(n=214 / 471)$ and a long-term mortality rate of only $30.1 \%(n=142 / 471)$.

Conclusions: Care limitations significantly influenced the validity of common prognostication models resulting in overestimation of poor outcome. The max- $\mathrm{ICH}$ score demonstrated increased predictive validity with minimized confounding by care limitations, making it a useful tool for severity assessment in ICH patients. Neurology ${ }^{\circledR}$ 2017;89:423-431

\section{GLOSSARY}

AUC = area under the curve; $\mathbf{C I}=$ confidence interval; $\mathbf{D N R}=$ do not resuscitate; $\mathbf{E C L}=$ early care limitations; $\mathbf{G C S}=$ Glasgow Coma Scale; ICH = intracerebral hemorrhage; IVH = intraventricular hemorrhage; $\mathbf{M I C H}=$ modified intracerebral hemorrhage score; $\mathbf{m R S}=$ modified Rankin Scale; NIHSS = NIH Stroke Scale; OR = odds ratio; PPV = positive predictive value; $\mathbf{P S}=$ propensity score; $\mathbf{R O C}=$ receiver operating characteristic.

Over decades, case fatality and long-term outcome have remained unchanged in patients with intracerebral hemorrhage $(\mathrm{ICH}) .{ }^{1}$ Initial aggressive treatment may provide benefit, yet overall ICH still lacks effective therapies to improve outcome. ${ }^{2-4}$ End-of-life decisions therefore play an important role in $\mathrm{ICH}$ management. ${ }^{5}$ To ensure best possible care, treating physicians, relatives, and patients need accurate information regarding the severity of $\mathrm{ICH}$ and its predicted outcome. $^{6}$

The most important limitation of existing prognostication models_-stated by American Heart Association/American Stroke Association guidelines-relates to care limitations, i.e., withholding/withdrawal of medical support, do-not-resuscitate (DNR) orders, and comfort

Supplemental data at Neurology.org
From the Departments of Neurology (J.A.S., S.T.G., B.V., T.B., S.S., H.B.H., J.B.K.) and Neuroradiology (H.L., S.P.K.), University Hospital Erlangen, Germany.

Go to Neurology.org for full disclosures. Funding information and disclosures deemed relevant by the authors, if any, are provided at the end of the article. 
care. ${ }^{5,7,8}$ The developers of the most commonly used grading scale-the ICH scorerecently demonstrated that avoidance of early DNR orders resulted in substantially lower mortality than predicted. ${ }^{8}$ In addition, a prospective study questioned the validity of these models as physicians' early clinical judgment correlated more closely with functional outcome than prognostic scores.?

In routine management, most $\mathrm{ICH}$ patients receive early $(<24$ hours) care limitations (ECL) based on a perceived poor prognosis, potentially leading to a self-fulfilling prophecy. ${ }^{5,10}$ Hence, chance of recovery will be denied, perpetuating this dilemma. This highlights the need to reduce an unwanted variability of informal physician-based prognostication and the inaccuracy of models variably including patients with ECL. ${ }^{8,11,12}$ The benefits of aggressive care may be underappreciated as no model focuses exclusively on maximally treated patients. ${ }^{11,12}$ As a result, the true prognosis of functional long-term outcome remains uncertain. $^{5}$

Therefore, this study sought to characterize the influence of ECL on (1) outcome using propensity score matching and (2) validity of prognostication by analyses of prediction models in both maximally treated and overall $\mathrm{ICH}$ patients. Based on identified predictors in maximally treated patients, we aimed to develop a grading scale_-called the max-ICH score- to provide severity assessment for functional long-term outcome with minimized confounding by care limitations.

METHODS Patient selection. We extracted pertinent data for consecutive ICH patients treated at the Department of Neurology (University Hospital Erlangen, Germany) from our prospective institutional ICH registry over a 5-year study period (January 2007 December 2011). We excluded secondary ICH etiologies, i.e., arteriovenous malformations, tumor, trauma, or $\mathrm{ICH}$ after acute thrombolysis or with a platelet count $<50,000 / \mu \mathrm{L}$. Overall, 621 spontaneous ICH patients of central European descent were identified. Thirty-eight patients were excluded because they refused consent ( $n=6$ ), were lost to follow-up ( $n=18)$, or the aggressiveness of care could not be precisely evaluated $(\mathrm{n}=14)$, as described below. Altogether, 583 remained for final analyses.

Standard protocol approvals, registrations, and patient consents. The study was approved by the ethics committee and informed consent was obtained from patients or legal representatives.

Early care limitations. We defined ECL as care limitations actually employed during the first 24 hours after admission.
ECL consisted of withholding or withdrawal of potentially lifesustaining treatment and induction of comfort care measures with the expectation that the patient would die as a result. ${ }^{10,13}$ Life-sustaining treatment included mechanical ventilation, cardiopulmonary resuscitation, use of vasopressors, antibiotics, or intracranial surgery (i.e., placement of external ventricular drain or intraparenchymal intracranial pressure probes, hematoma evacuation). A written DNR order alone was not considered a care limitation. Any care limitations during the hospital stay as well as timing, reason, and mode were evaluated retrospectively by 2 independent physicians. In cases of inconsistencies, a second consensus analysis was carried out by a third investigator and if no consensus was reached the patient was excluded.

Data acquisition. Demographics, medical history, neuroradiologic data, and in-hospital measures were extracted, as previously described. ${ }^{14}$ In-hospital measures on admission included Glasgow Coma Scale (GCS), NIH Stroke Scale (NIHSS), brainstem affection represented by absent pupillary light reflex or corneal reflex in 1 or both eyes (after controlling for factors compromising reflex status), mechanical ventilation, type of hospital ward, and length of hospital stay. For a description of neuroradiologic data acquisition, see the e-Methods at Neurology.org. Mortality and functional outcome were recorded for 1 year of follow-up, determined as short-term (3-month) and long-term (12-month) outcome. Functional outcome was evaluated by modified Rankin Scale (mRS) and dichotomized as favorable (mRS 0-3) or unfavorable (mRS 4-6), as previously described. ${ }^{15}$

\section{Prognostic models: ICH score and modified ICH score.} As most ICH scoring systems target short-term outcome, the literature review identified only 2 models (ICH score and modified ICH score $[\mathrm{MICH}]$ ) developed or validated for 12-month outcome prognostication. ${ }^{7,16-22}$ The ICH score was calculated as previously described, summarizing points for GCS $(15-13=0$, $12-5=1,4-3=2)$, ICH volume $\left(<30 \mathrm{~cm}^{3}=0, \geq 30 \mathrm{~cm}^{3}=1\right)$, presence of intraventricular hemorrhage (IVH) $($ no $=0$, yes $=1)$, infratentorial origin (no $=0$, yes $=1)$, and patient age $(<80=0$, $\geq 80=1){ }^{7}$ The predicted short-term mortality for each patient was determined according to the patient's ICH score using the corresponding observed mortality rate from the ICH score development cohort (ICH score 0, 0\%; 1, 13\%; 2, 26\%; 3, 72\%; 4, $97 \% ; 5,100 \%){ }^{7.8}$ The predicted mortality for the present cohort was calculated as the average of the predicted mortality across patients, as described previously. ${ }^{7.8}$ Contrary to the original ICH score cohort, 1 single patient of our study had an ICH score of 6 , therefore lacking a prior observed mortality rate. ${ }^{7}$ Only for graphical comparison of short-term mortality rates between cohorts, we classified this patient into the next lower category $(5 ; 100 \%)$. The $\mathrm{MICH}$ was calculated by summarizing points for GCS (15-13 = $0,12-5=1,4-3=2)$, ICH volume $(\leq 20=0,21-50=1, \geq 51$ $=2$ ), and presence of IVH or hydrocephalus (no $=0$, yes $=1$ ), as appropriate. ${ }^{17}$

Statistical analysis. Statistical analyses were performed with SPSS version 20.0 (SPSS Inc., Chicago, IL) and R version 2.12.1. Statistical tests were 2-sided, significance level was set at $\alpha=0.05$, multiple comparisons were corrected by Bonferroni method (type I error). Missing data were handled as complete case analyses. Kolmogorov-Smirnov test was used to determine data distribution. Normally distributed data are shown as mean \pm $\mathrm{SD}$, compared using Student $t$ test; non-normally distributed data as median and interquartile range, compared using the MannWhitney $U$ test. Pearson $\chi^{2}$ and Fisher exact tests were applied to compare frequency distributions of categorized variables. We utilized explorative analyses to identify factors associated with 
Table 1 Characteristics of early care limitations patients vs maximally treated patients

\begin{tabular}{|c|c|c|c|}
\hline Intracerebral hemorrhage $(n=583)$ & Early care limitations ( $n=112$ ) & Maximal treatment $(n=471)$ & $p$ Value \\
\hline Age, y & $75 \pm 11$ & $70 \pm 12$ & $<0.001$ \\
\hline Female sex & $64(57.1)$ & $214(45.4)$ & $0.03^{a}$ \\
\hline \multicolumn{4}{|l|}{ Medical history } \\
\hline Hypertension & $83(74.1)$ & $385(81.7)$ & 0.07 \\
\hline Diabetes mellitus & 22 (19.6) & $127(27.0)$ & 0.11 \\
\hline Dyslipidemia & 22 (19.6) & 152 (32.3) & $0.01^{a}$ \\
\hline Prior ischemic stroke & $24(21.4)$ & 103 (21.9) & 0.92 \\
\hline Prior ICH & $5(4.5)$ & 37 (7.9) & 0.21 \\
\hline Antiplatelet medication & 37 (33.0) & $137(29.1)$ & 0.41 \\
\hline Oral anticoagulation & $18(16.1)$ & $90(19.1)$ & 0.46 \\
\hline \multicolumn{4}{|l|}{ Admission status } \\
\hline Glasgow Coma Scale & $4(3-7)$ & $13(10-15)$ & $<0.001$ \\
\hline NIHSS & 29 (24-32) & $11(5-19)$ & $<0.001$ \\
\hline Brainstem affection & $75(67.0)$ & $40(8.5)$ & $<0.001$ \\
\hline $\mathrm{ICH}$ score & $4(3-4)$ & $1(0-2)$ & $<0.001$ \\
\hline \multicolumn{4}{|l|}{ Neuroradiologic data } \\
\hline \multicolumn{4}{|l|}{ Location } \\
\hline Lobar & $50(44.6)$ & $199(42.3)$ & 0.65 \\
\hline Deep & 44 (39.3) & $223(47.3)$ & 0.12 \\
\hline Cerebellar & $7(6.3)$ & $28(5.9)$ & 0.92 \\
\hline Brainstem & $11(9.8)$ & $21(4.5)$ & $0.03^{a}$ \\
\hline $\mathrm{ICH}$ volume, $\mathrm{cm}^{3}$ & $76.3 \pm 57.1$ & $22.5 \pm 26.0$ & $<0.001$ \\
\hline Intraventricular hemorrhage & $92(82.1)$ & 213 (45.2) & $<0.001$ \\
\hline Graeb score & $5(2-8)$ & $0(0-3)$ & $<0.001$ \\
\hline \multicolumn{4}{|l|}{ In-hospital measures } \\
\hline ICP monitoring & $11(9.8)$ & $142(30.1)$ & $<0.001$ \\
\hline Hematoma evacuation & $2(1.8)$ & $28(5.9)$ & 0.07 \\
\hline Admitted to NCU & $71(63.4)$ & $471(100.0)$ & $<0.001$ \\
\hline Admitted to general ward & $41(36.6)$ & $0(0.0)$ & $<0.001$ \\
\hline Length of hospital stay, $d$ & $1(1-2)$ & $12(7-19)$ & $<0.001$ \\
\hline
\end{tabular}

Abbreviations: $\mathrm{ICH}=$ intracerebral hemorrhage; ICP = intracranial pressure; $\mathrm{NCU}=$ neurocritical care unit; $\mathrm{NIHSS}=\mathrm{NIH}$ Stroke Scale.

ICP monitoring consisted of external ventricular drains or intraparenchymal probes. Values are mean \pm SD, $n(\%)$, or median (interquartile range; 25 th-75th percentile).

${ }^{\text {a }}$ Not significant after Bonferroni correction.

ECL by stepwise forward multivariable logistic regression models. To reach homogenous measurement levels and to obtain better comparability of odds ratio (OR) strengths of ECL-associated factors, metric measures were transformed into the same categorical variables according to ICH score determination. Further, we used propensity score (PS) matching to depict the theoretical outcome of ECL patients if provided maximal treatment. We compared the prognostic validity of the ICH score between the entire cohort and maximally treated patients (i.e., non-ECL patients) using receiver operating characteristic (ROC) curves and Youden J statistics. ${ }^{23}$ Areas under the curves (AUC) were analyzed using the technique of Hanley and McNeil. ${ }^{24}$ Positive predictive values (PPV) for mortality and functional outcome were compared using $\chi^{2}$ between test computations and considered not equal if the observed value exceeded the critical value of 3.84 . The max-ICH score was developed, analyzed, and compared to the $\mathrm{ICH}$ score and $\mathrm{MICH}{ }^{7,17}$ For further statistical details, see the e-Methods.

RESULTS This cohort included $583 \mathrm{ICH}$ patients and baseline characteristics were as follows: age 71

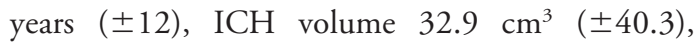
GCS 13 (5-15), infratentorial ICH 67 (11.5\%), IVH 305 (52.3\%), not significantly differing from patients $(\mathrm{n}=38)$ excluded from analysis. The present cohort was comparable to study populations used for ICH score development and long-term 
functional outcome validation regarding $\mathrm{ICH}$ severity, all with median ICH scores of 2 (1-3); for comparison, see table e-1.7,16

Early care limitations ( $<\mathbf{2 4}$ hours). The prevalence of ECL was $19.2 \%(\mathrm{n}=112 / 583)$; after initial diagnosis, $83.9 \%(\mathrm{n}=94 / 112)$ of patients did not receive further therapy (withholding) and therapy was withdrawn within the first 24 hours in $16.1 \%(n=18 /$ 112). All patients with identified ECL died after a median hospital stay of 1 (1-2) days. A total of 156 care limitations were recorded and $71.8 \%(\mathrm{n}=$ $112 / 156)$ were judged as ECL. Of all patients, only $3.1 \%(\mathrm{n}=18 / 583)$ received care limitations within 24-72 hours and 4.5\% ( $n=26 / 583)$ during the subsequent clinical course. The median length of hospital stay for patients with delayed care limitations (n $=44 / 583)$ was 7 (3-12) days and reasons involved hematoma enlargement $(n=23)$, patient's presumed will $(\mathrm{n}=11)$, or clinical deterioration or lack of improvement $(\mathrm{n}=10)$. We therefore consider the investigated cohort of no ECL as maximally treated patients $(\mathrm{n}=471)$.

Table 1 compares baseline characteristics between patients with ECL and maximal treatment, showing significant associations for measures integrated in the ICH score. The seldom-assessed measure brainstem affection (absent brainstem reflexes) was almost 8 times more frequent in ECL patients and showed the strongest link to ECL upon multivariable modeling (OR 12.79, confidence interval [CI] 6.00-27.27, $p<0.01$ ), followed by GCS (OR 8.81, CI 3.10 $25.06, p<0.01$ ), age (OR 7.44, CI 3.42-16.20, $p<$ 0.01 ) and ICH volume (OR 3.98, CI 2.07-7.66, $p<$ $0.01)$. ECL prevalence and patient characteristics

\section{Figure 1 Comparison of mortality rates}

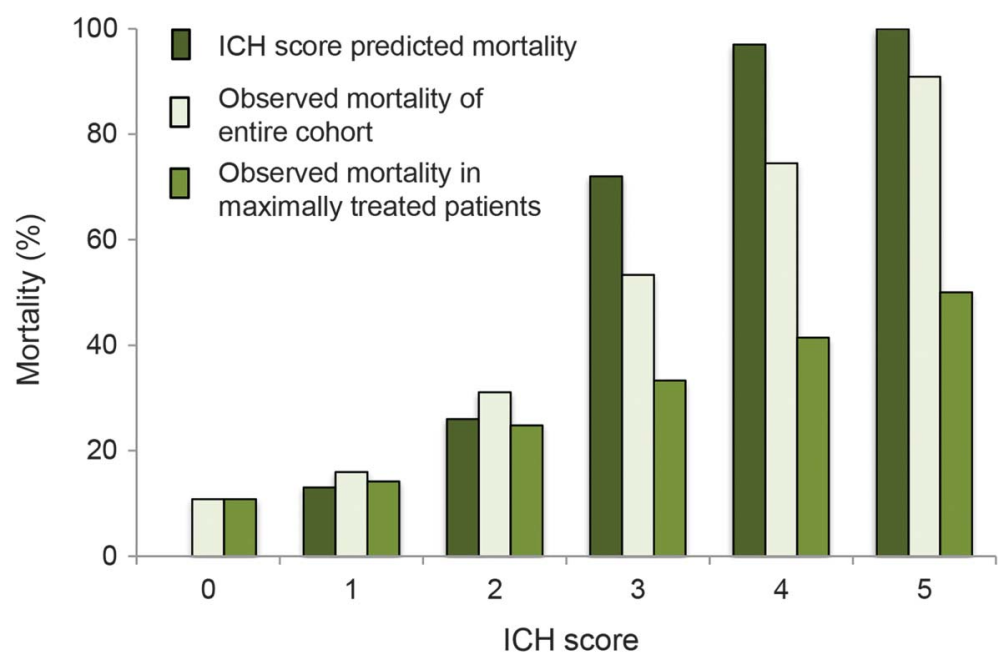

Observed short-term mortality rate in the entire intracerebral hemorrhage (ICH) cohort $(n=$ $583)$ and in maximally treated patients $(n=471)$ in contrast to predicted short-term mortality rate by the $\mathrm{ICH}$ score. were similar compared to previous investigations (table e-2).

To investigate the theoretical benefit of aggressive care, we conducted a rigorous PS matching to balance patients with ECL to maximally treated patients. The successful matching procedure-statistically consistent outcome predictors between ECL and nonECL patients (table e-3) - showed that half (50.7\%, $\mathrm{n}=35 / 69$ ) of the maximally treated patients actually survived and even achieved favorable long-term outcome in $18.8 \%(\mathrm{n}=13 / 69)$. Thus, we theoretically may have refused patients' chance of survival in at least $37.7 \%(n=26 / 69)$, as in $13 \%(n=9 / 69)$ of matched ECL cases further maximal treatment was dissented by patient's will expressed by advance care directives.

Validity of outcome prediction. We investigated the influence of ECL on outcome prognostication using the ICH score and compared predicted with actual short-term mortality rates: (1) predicted, (2) entire cohort, (3) maximally treated cohort (figure 1). For maximally treated patients, the ICH score predicted a mortality rate of $29 \%$ ( $n=135 / 471)$, yet the observed mortality rate was only $21 \%(n=99 / 471)$. This overestimation increased with rising $\mathrm{ICH}$ scores; i.e., predicted mortality rate of $97.3 \%$ for patients with a score $\geq 4$ compared to observed mortality rate of only $42.2 \%$. The ICH score was strongly linked to applied ECL as 3 of 4 parameters were independently associated and ROC analyses showed a stronger association for the ICH score with ECL (AUC 0.88, CI 0.85-0.91, $p<0.01)$ than with actual short-term mortality (AUC 0.80, CI 0.76-0.83, $p<0.01$ ).

In figure 2, we present ROC analyses comparing the prognostic performances of the ICH score in both cohorts: (1) entire cohort vs (2) maximally treated patients. The predictive validity for mortality and functional outcome decreased in maximally treated patients (short-term mortality: AUC 0.80, CI 0.760.83 vs AUC 0.67, CI 0.61-0.73, long-term functional outcome: AUC 0.79, CI $0.75-0.82$ vs AUC 0.72 , CI 0.68-0.75, both $p<0.05$ ). Accordingly, we observed decreased precision (short-term mortality: $\mathrm{PPV}=67.0 \%$ vs $\mathrm{PPV}=37.0 \%$, long-term functional outcome: $\mathrm{PPV}=83.1 \%$ vs $\mathrm{PPV}=74.7 \%$, both $p<0.05$ ) at optimal cutoffs. Application of the ICH score's precision calculated for the entire cohort led to overestimation of poor outcome in maximally treated patients, resulting in a net difference of $44.8 \%$ for short-term mortality and $10.1 \%$ for long-term unfavorable outcome.

Novel severity assessment: The max-ICH score. To account for the observed inaccuracy and to improve severity assessment, we created a grading scale-the maxICH score-containing all measures independently 

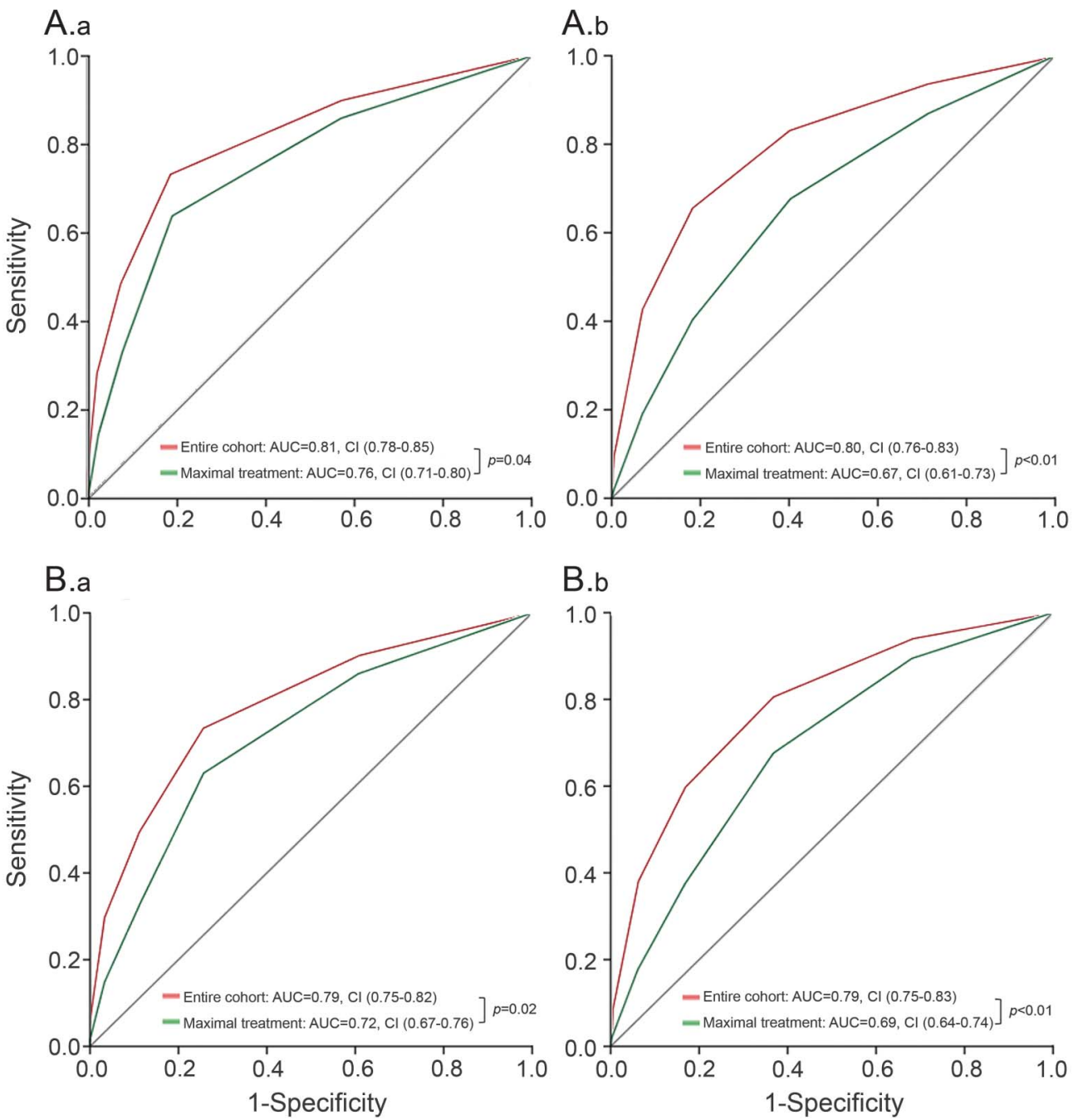

Receiver operating characteristic curves for the association of the ICH score with short-term (A.a) unfavorable functional outcome and (A.b) mortality as well as long-term (B.a) unfavorable functional outcome and (B.b) mortality for the entire cohort and in patients with maximal treatment. $\mathrm{AUC}=$ area under the curve; $\mathrm{Cl}=$ confidence interval; $\mathrm{ICH}=$ intracerebral hemorrhage.

associated with unfavorable long-term outcome (mRS 4-6) upon multivariable modeling in maximally treated patients, i.e., ICH volume (OR 1.02, CI 1.01$1.03, p<0.01$ ), age (OR 1.08, CI 1.06-1.10, $p<$ 0.01 ), NIHSS (OR 1.11, CI 1.08-1.14, $p<0.01$ ), IVH (OR 1.66, CI 1.03-2.67, $p=0.04$ ), and oral anticoagulation (OR 2.08, CI 1.18-3.68, $p=$ 0.01) (table e-4). Measures such as infratentorial ICH, GCS, and brainstem affection failed to improve the model's statistical strength or did not reach significance. Point assignment was based on identified statistical potency at optimal cutoffs (figure e-1). Table 2 demonstrates the point assignment for each of the 6 max-ICH score components with a total score ranging from 0 to 10 . We compared the max- $\mathrm{ICH}$ score with the $\mathrm{ICH}$ score and $\mathrm{MICH}$ (figure 3A) - the only scores validated for long-term outcome assessment-demonstrating superior predictive validity for both long-term functional outcome (AUC 0.81, CI $0.77-0.85, p<0.01$ ) and mortality (AUC 0.77, CI $0.72-0.81, p<0.01$ ). The same holds true for prognostication in the entire cohort (figure e-2). Analyzing the influence of ECL on the max-ICH score, we found no confounding of outcome prediction (functional outcome, entire cohort: AUC 0.85, CI $0.82-0.88$ vs maximal treatment: AUC 0.81, CI 0.77-0.85, $p=$ 0.11 ). In figure $3 \mathrm{~B}$, we present long-term outcome data in maximally treated patients, documenting that almost half $(45.4 \%, \mathrm{n}=214 / 471)$ of the patients reached favorable functional outcome and only $30.1 \%$ ( $\mathrm{n}=$ 142/471) died during 12-month follow-up. Figure 3B also provides outcome stratification according to the max-ICH score, indicating point estimates for mortality and favorable functional outcome with corresponding CIs. To investigate prognostication in patients potentially prone for care limitations, we 


\section{Table 2 Determination of the max-ICH score}

Component

Points

NIHSS

0-6

0

7-13

14-20

2

$\geq 21$

Age, y

$\leq 69$

70-74

75-79

$\geq 80$

Intraventricular hemorrhage

No

Yes

Oral anticoagulation

No

0

Yes

Lobar ICH volume, $\mathrm{cm}^{3}$

$<30$

0

$\geq 30$

Nonlobar ICH volume, $\mathrm{cm}^{3}$

$<10$

$\geq 10$

Total max-ICH score
DISCUSSION Almost one-fifth of ICH patients received ECL and analyses demonstrated the potential of false poor outcome attribution resulting in a self-fulfilling prophecy. ECL seemed to influence the predictive validity of current prognostication tools especially in maximally treated patients, leading to overestimation of mortality and unfavorable functional outcome. We developed a simple grading scale-the max-ICH score-which allows severity assessment less biased by care limitations and that more accurately delineates the potential of aggressive care in ICH. Among maximally treated patients, more than 2-thirds survived and almost every second patient reached favorable functional outcome.

A recent nationwide survey conducted in the United States demonstrated that physicians vary substantially in ICH prognostic estimates and treatment recommendations, which may lead to profound differences in life and death decision-making. ${ }^{12}$ Theoretically, prognostication models provide point estimates to reduce this unwanted variability. ${ }^{12}$ Hemphill et al. ${ }^{7,16}$ developed the ICH score as a simple grading scale for ICH in 2001 and it has since been validated for mortality and functional long-term outcome. The developers of the ICH score recently reported in a prospective multicenter study on moderately to severely $(\mathrm{GCS} \leq 12)$ affected ICH patients that the observed short-term mortality was lower than predicted under full medical support. ${ }^{8}$ We confirmed the diverging short-term mortality under maximal treatment in all $\mathrm{ICH}$ patients irrespective of neurologic status. We further documented relevant confounding effects introduced by ECL, resulting in a reduced predictive validity of conventional grading scales on functional outcome. In maximally treated patients, the ICH score fulfills the main purpose it was designed for, i.e., stratifying the risk of short-term mortality, yet our data demonstrated increased inaccuracy with overestimation of poor outcome. As suggested by PS-matched analyses, refusal of aggressive therapy possibly resulted in self-fulfilling prophecies diminishing chance of potential recovery. ${ }^{8,26}$ This seems to have influenced prognostic model $^{25}$ and such predicted poor outcome runs the risk to become self-perpetuating, emphasizing the need for a less biased approach of severity assessment in $\mathrm{ICH}$.

We developed the max-ICH score to improve risk stratification and to evaluate the influence of maximal treatment on functional long-term outcome. The integrated measures are routinely assessed during acute ICH management and incorporate initial imaging data to allow for a quick severity assessment on admission. The max-ICH score ranges from 0 to 10 and chances of survival and favorable functional 

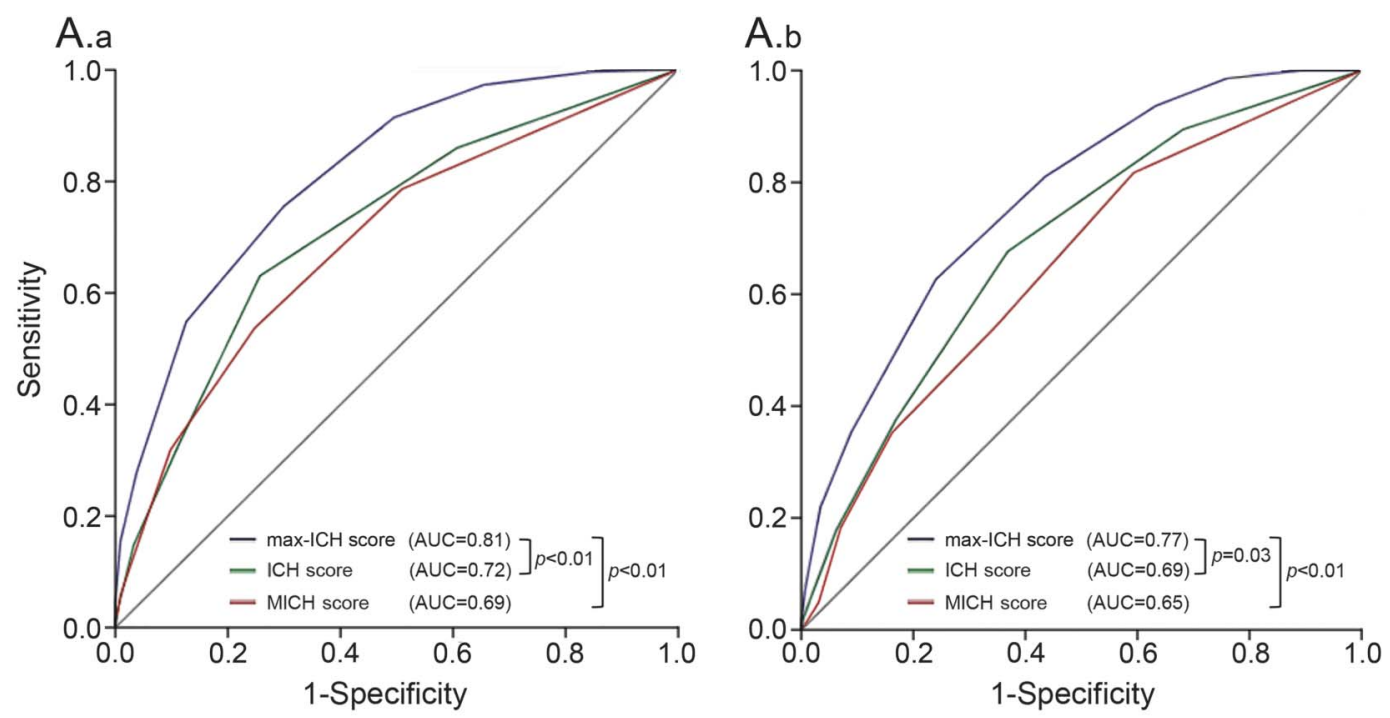

B

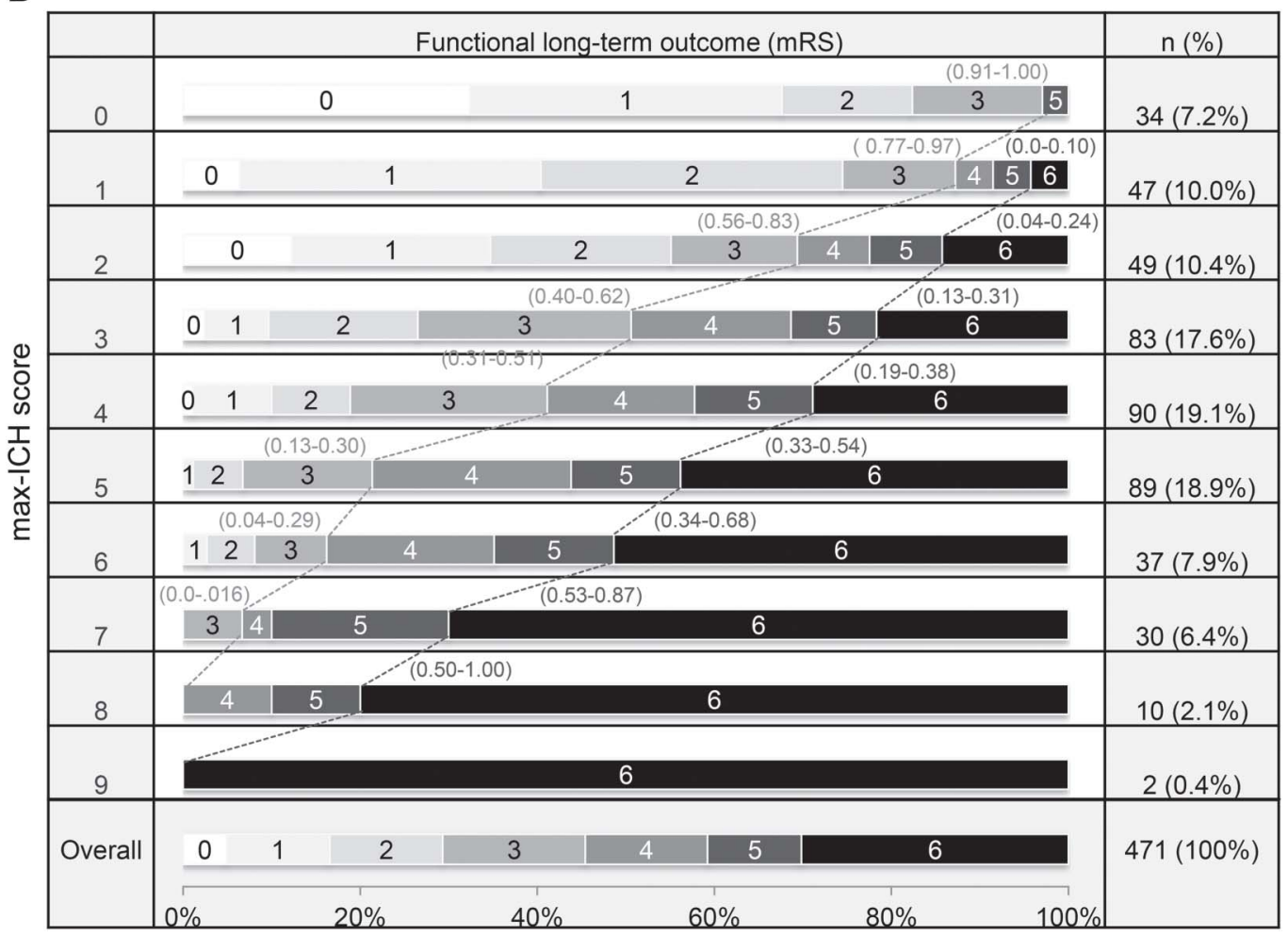

(A) Receiver operating characteristic curves for the association of the max-ICH score, the ICH score, and the modified intracerebral hemorrhage score (MICH) with (A.a) unfavorable functional long-term outcome and (A.b) long-term mortality. (B) Functional long-term outcome evaluated by modified Rankin Scale (mRS) in relation to max-ICH score classification providing point estimates for mortality and favorable functional outcome (mRS 0-3) with corresponding confidence intervals. $\mathrm{AUC}=$ area under the curve; $\mathrm{ICH}=$ intracerebral hemorrhage.

long-term outcome decrease with rising score values. Yet point estimates are provided and remain relevant up to the top end of the score as the important proportion of patients prone to false poor outcome attribution is significantly decreased. In patients with a score above 7 , none reached favorable functional outcome, while in patients with a score below 2 , more than 9 out of 10 achieved functional independence at
1 year. Although the max-ICH score was predictive of outcome, exact prognostication for an individual is not possible. ${ }^{7,8}$ The aim of the present score is not to identify patients in whom further care is futile but to show in which patients aggressive care is warranted and potentially beneficial. Despite the remaining uncertainty of cohort-based models, prognostic information is needed to support shared decision-making 
early in the setting of $\mathrm{ICH}^{8,25}$ and needs to be as accurate, unbiased, and invariable as possible.

The present study has several strengths, as outcome data were prospectively collected until 12month follow-up and rigorous statistical tools have been applied. We consider the present cohort to be representative and valid for outcome prediction, as analysis of the ICH score's predicted short-term mortality with a pooled analysis of 9 cohorts including 3,819 participants worldwide confirmed comparability (present study: AUC 0.80 , CI $0.76-0.83$ vs pooled analysis: AUC 0.80 , CI $0.77-0.85^{27}$ ). Moreover, ECL determination seems to be valid as we confirmed prevalence and characteristics (i.e., older age, lower GCS, greater ICH volume) compared to related studies. ${ }^{10,26,28,29}$

Shortcomings limit the interpretation of our findings. First, the study was monocentric and specific center characteristics may not be transferable. Decisions to limit maximal care were based on estimation by attending physicians in accordance with the patient's will or presumed will. Second, although other measures were collected prospectively, ECL were scored retrospectively, possibly attenuating data quality. Third, exclusion of ECL patients could have led to selection bias, as patients with a theoretic potential for recovery may have been falsely classified. However, validity of outcome prediction was based on ICH scores, which likely provided protection against bias due to severity adjustments. ${ }^{8}$ As the max-ICH score was developed in a selected cohort (i.e., maximally treated patients), its application to all $\mathrm{ICH}$ patients should be carefully evaluated. Nevertheless, we provide a model based on predictors identified in a cohort with minimized confounding due to care limitations, which led to higher predictive validity not only in patients chosen for maximal treatment but also in the entire cohort of ICH patients. We further cannot exclude prehospital transferal effects. Finally, despite exclusion of ECL patients, outcome predictors may be affected by delayed care limitations $(7.5 \%, \mathrm{n}=44 / 583)$. However, this small number of patients received maximal treatment unsuccessfully and did not influence overall prognostication (data not shown). Furthermore, exclusion of all patients with care limitations during the hospital stay seems unreasonable as almost all in-hospital deaths receive some form of care limitation in neurocritical care. ${ }^{30}$

Despite the inherent limitations of cohort studies, we provide evidence that commonly used prognostication models in ICH may be influenced by care limitations, resulting in reduced validity and overestimation of poor outcome. Theoretically, ECL may have denied some patients' chance of recovery, as observed long-term outcome in maximally treated patients was more favorable than perceived. We introduced a grading scale developed in maximally treated patients, allowing for severity assessment with minimized confounding by care limitations. The max-ICH score may improve multimodal assessment of ICH patients, may aid interphysician communication and clinical research investigations, and may help to evaluate long-term treatment and recovery goals. ${ }^{8}$ However, validation through improved study design seems strongly warranted.

\section{AUTHOR CONTRIBUTIONS}

Jochen Sembill: study concept, acquisition, analysis and interpretation of data. Stefan Gerner: data acquisition, critical revision of manuscript for intellectual content. Bastian Volbers: data acquisition, critical revision of manuscript for intellectual content. Tobias Bobinger: data acquisition, critical revision of manuscript for intellectual content. Hannes Lücking: data acquisition, critical revision of manuscript for intellectual content. Stephan Kloska: data acquisition, critical revision of manuscript for intellectual content. Stefan Schwab: study concept, critical revision of manuscript for intellectual content. Hagen Huttner: study concept, interpretation of data, study supervision. Joji Kuramatsu: study concept, acquisition, analysis, interpretation of data, study supervision.

\section{STUDY FUNDING}

No targeted funding reported.

\section{DISCLOSURE}

J. Sembill, S. Gerner, B. Volbers, T. Bobinger, H. Lücking, S. Kloska, and S. Schwab report no disclosures relevant to the manuscript. H. Huttner: speaker honoraria and travel grants from Boehringer Ingelheim and Bayer Healthcare J. Kuramatsu: travel grants from EMCools, Otsuka, and Boehringer Ingelheim, and speaker honoraria from Otsuka. Go to Neurology.org for full disclosures.

Received September 9, 2016. Accepted in final form March 27, 2017.

\section{REFERENCES}

1. Zahuranec DB, Lisabeth LD, Sanchez BN, et al. Intracerebral hemorrhage mortality is not changing despite declining incidence. Neurology 2014;82:2180-2186.

2. Kuramatsu JB, Gerner ST, Schellinger PD, et al. Anticoagulant reversal, blood pressure levels, and anticoagulant resumption in patients with anticoagulation-related intracerebral hemorrhage. JAMA 2015;313:824-836.

3. Flaherty ML, Haverbusch M, Sekar P, et al. Long-term mortality after intracerebral hemorrhage. Neurology 2006; 66:1182-1186

4. Anderson CS, Heeley E, Huang Y, et al; INTERACT2 Investigators. Rapid blood-pressure lowering in patients with acute intracerebral hemorrhage. $N$ Engl J Med 2013;368:2355-2365.

5. Holloway RG, Arnold RM, Creutzfeldt CJ, et al; American Heart Association Stroke Council, Council on Cardiovascular and Stroke Nursing, and Council on Clinical Cardiology. Palliative and end-of-life care in stroke: a statement for healthcare professionals from the American Heart Association/American Stroke Association. Stroke 2014;45: 1887-1916.

6. Geurts M, Macleod MR, van Thiel GJ, van Gijn J, Kappelle LJ, van der Worp HB. End-of-life decisions in patients with severe acute brain injury. Lancet Neurol 2014; 13:515-524. 
7. Hemphill JC III, Bonovich DC, Besmertis L, Manley GT, Johnston SC. The ICH score: a simple, reliable grading scale for intracerebral hemorrhage. Stroke 2001;32: 891-897.

8. Morgenstern LB, Zahuranec DB, Sanchez BN, et al. Full medical support for intracerebral hemorrhage. Neurology 2015;84:1739-1744.

9. Hwang DY, Dell CA, Sparks MJ, et al. Clinician judgment vs formal scales for predicting intracerebral hemorrhage outcomes. Neurology 2016;86:126-133.

10. Zahuranec DB, Brown DL, Lisabeth LD, et al. Early care limitations independently predict mortality after intracerebral hemorrhage. Neurology 2007;68:1651-1657.

11. Hemphill JC III, Greenberg SM, Anderson CS, et al; American Heart Association Stroke Council; Council on Cardiovascular and Stroke Nursing; Council on Clinical Cardiology. Guidelines for the management of spontaneous intracerebral hemorrhage: a guideline for healthcare professionals from the American Heart Association/American Stroke Association. Stroke 2015;46:2032-2060.

12. Zahuranec DB, Fagerlin A, Sanchez BN, et al. Variability in physician prognosis and recommendations after intracerebral hemorrhage. Neurology 2016;86:1864-1871.

13. Smedira NG, Evans BH, Grais LS, et al. Withholding and withdrawal of life support from the critically ill. N Engl J Med 1990;322:309-315.

14. Kuramatsu JB, Bobinger T, Volbers B, et al. Hyponatremia is an independent predictor of in-hospital mortality in spontaneous intracerebral hemorrhage. Stroke 2014;45: 1285-1291.

15. Kuramatsu JB, Gerner ST, Lucking H, et al. Anemia is an independent prognostic factor in intracerebral hemorrhage: an observational cohort study. Crit Care 2013;17:R148.

16. Hemphill JC III, Farrant M, Neill TA Jr. Prospective validation of the $\mathrm{ICH}$ score for 12-month functional outcome. Neurology 2009;73:1088-1094.

17. Cho DY, Chen CC, Lee WY, Lee HC, Ho LH. A new modified intracerebral hemorrhage score for treatment decisions in basal ganglia hemorrhage: a randomized trial. Crit Care Med 2008;36:2151-2156.

18. Cheung RT, Zou LY. Use of the original, modified, or new intracerebral hemorrhage score to predict mortality and morbidity after intracerebral hemorrhage. Stroke 2003;34:1717-1722.
19. Chuang YC, Chen YM, Peng SK, Peng SY. Risk stratification for predicting 30-day mortality of intracerebral hemorrhage. Int J Qual Health Care 2009;21:441-447.

20. Ruiz-Sandoval JL, Chiquete E, Romero-Vargas S, PadillaMartinez JJ, Gonzalez-Cornejo S. Grading scale for prediction of outcome in primary intracerebral hemorrhages. Stroke 2007;38:1641-1644.

21. Godoy DA, Pinero G, Di Napoli M. Predicting mortality in spontaneous intracerebral hemorrhage: can modification to original score improve the prediction? Stroke 2006;37: 1038-1044.

22. Rost NS, Smith EE, Chang Y, et al. Prediction of functional outcome in patients with primary intracerebral hemorrhage: the FUNC score. Stroke 2008;39:2304-2309.

23. Youden WJ. Index for rating diagnostic tests. Cancer 1950;3:32-35.

24. Hanley JA, McNeil BJ. The meaning and use of the area under a receiver operating characteristic (ROC) curve. Radiology 1982;143:29-36.

25. Hemphill JC III, White DB. Clinical nihilism in neuroemergencies. Emerg Med Clin North Am 2009;27:27-37, vii-viii.

26. Becker KJ, Baxter AB, Cohen WA, et al. Withdrawal of support in intracerebral hemorrhage may lead to selffulfilling prophecies. Neurology 2001;56:766-772.

27. Mattishent K, Kwok CS, Ashkir L, Pelpola K, Myint PK, Loke YK. Prognostic tools for early mortality in hemorrhagic stroke: systematic review and meta-analysis. J Clin Neurol 2015;11:339-348.

28. Nakagawa K, Vento MA, Seto TB, et al. Sex differences in the use of early do-not-resuscitate orders after intracerebral hemorrhage. Stroke 2013;44:3229-3231.

29. Silvennoinen K, Meretoja A, Strbian D, Putaala J, Kaste M, Tatlisumak T. Do-not-resuscitate (DNR) orders in patients with intracerebral hemorrhage. Int $\mathrm{J}$ Stroke 2014;9:53-58.

30. Verkade MA, Epker JL, Nieuwenhoff MD, Bakker J, Kompanje EJ. Withdrawal of life-sustaining treatment in a mixed intensive care unit: most common in patients with catastropic brain injury. Neurocrit Care 2012;16:130135.

31. Falcone GJ, Biffi A, Brouwers HB, et al. Predictors of hematoma volume in deep and lobar supratentorial intracerebral hemorrhage. JAMA Neurol 2013;70:988-994.

\section{Subspecialty Alerts by E-mail!}

Customize your online journal experience by signing up for e-mail alerts related to your subspecialty or area of interest. Access this free service by visiting Neurology.org/site/subscriptions/etoc.xhtml or click on the "E-mail Alerts" link on the home page. An extensive list of subspecialties, methods, and study design choices will be available for you to choose from—allowing you priority alerts to cutting-edge research in your field! 


\section{Neurology}

Severity assessment in maximally treated ICH patients: The max-ICH score Jochen A. Sembill, Stefan T. Gerner, Bastian Volbers, et al.

Neurology 2017;89;423-431 Published Online before print July 5, 2017

DOI 10.1212/WNL.0000000000004174

This information is current as of July 5, 2017

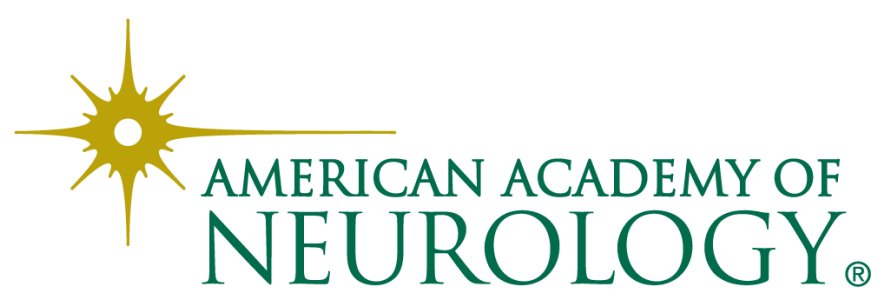




\section{Updated Information \& Services}

\section{Supplementary Material}

\section{References}

Citations

Subspecialty Collections

\section{Permissions \& Licensing}

\section{Reprints}

including high resolution figures, can be found at: http://n.neurology.org/content/89/5/423.full.html

Supplementary material can be found at: http://n.neurology.org/content/suppl/2017/07/05/WNL.0000000000004 174.DC1

http://n.neurology.org/content/suppl/2017/07/05/WNL.0000000000004 174.DC2

This article cites 31 articles, 16 of which you can access for free at: http://n.neurology.org/content/89/5/423.full.html\#\#ref-list-1

This article has been cited by 1 HighWire-hosted articles: http://n.neurology.org/content/89/5/423.full.html\#\#otherarticles

This article, along with others on similar topics, appears in the following collection(s):

\section{All Cerebrovascular disease/Stroke}

http://n.neurology.org//cgi/collection/all_cerebrovascular_disease_strok e

Intracerebral hemorrhage

http://n.neurology.org//cgi/collection/intracerebral_hemorrhage

Other cerebrovascular disease/ Stroke

http://n.neurology.org//cgi/collection/other_cerebrovascular_disease_s troke

\section{Prognosis}

http://n.neurology.org//cgi/collection/prognosis

Information about reproducing this article in parts (figures,tables) or in its entirety can be found online at:

http://n.neurology.org/misc/about.xhtml\#permissions

Information about ordering reprints can be found online:

http://n.neurology.org/misc/addir.xhtml\#reprintsus

Neurology ${ }^{\circledR}$ is the official journal of the American Academy of Neurology. Published continuously since 1951, it is now a weekly with 48 issues per year. Copyright @ 2017 American Academy of Neurology. All rights reserved. Print ISSN: 0028-3878. Online ISSN: 1526-632X.

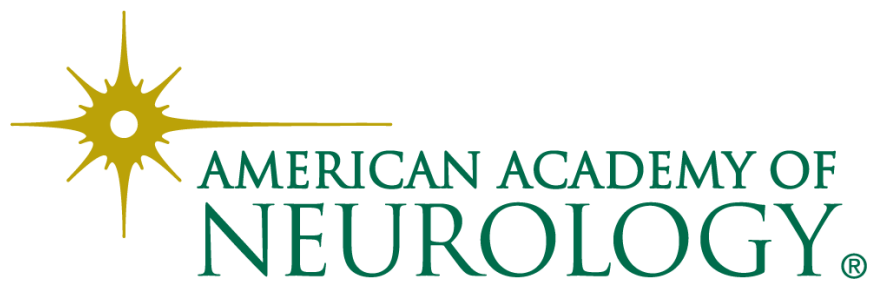

